\title{
CERTAIN HOMOMORPHISMS OF THE LATTICE OF VARIETIES OF COMPLETELY SIMPLE SEMIGROUPS
}

\author{
MARIO PETRICH and NORMAN R. REILLY
}

(Received 30 November 1981; revised 15 November 1982)

Communicated by T. E. Hall

\begin{abstract}
Completely simple semigroups form a variety, $\mathrm{CS}$, of algebras with the operations of multiplication and inversion. It is known that the mapping $\mathscr{V} \rightarrow(\mathfrak{V} \cap \mathcal{S}, \mathscr{V} \vee \mathcal{G})$, where $\mathcal{G}$ is the variety of all groups, is an isomorphism of the lattice $\mathcal{L}(\mathcal{S S}$ ) of all subvarieties of $\mathcal{S}$ onto a subdirect product of the lattice of subvarieties of $\mathcal{G}$ and the interval $[\mathcal{G}, \mathcal{C S}$. We consider embeddings of $\mathcal{L}(\mathcal{C S})$ into certain direct products on the above pattern with rectangular bands, rectangular groups and central completely simple semigroups in place of groups.
\end{abstract}

1980 Mathematics subject classification (Amer. Math. Soc.): 20 M 05, 20 M 07.

\section{Introduction}

Completely simple semigroups may be considered as algebras with the operation of multiplication and the unary operation of inversion. As such they form a variety $\mathcal{C S}$ determined by a small set of simple axioms. The construction of the free objects in $\mathcal{S}$ by Clifford [2] and Rasin [10] makes it possible to study the lattice $\mathcal{L}(\mathcal{S})$ of varieties of completely simple semigroups in some detail, see Rasin [10] and the authors [7], [8], [9]. This lattice turns out to be very complex, but some progress has been made and certain ideals have been completely determined. Another approach consists of considering certain endomorphisms of the lattice. Thus in [7], it was proved that the mapping $\mathfrak{V} \rightarrow(\mathfrak{V} \cap \mathcal{G}, \mathfrak{V} \vee \mathcal{G})$, where $\mathcal{G}$ is the variety of all groups, is a monomorphism of $\mathcal{L}(\mathcal{C S})$ onto a precisely characterized subdirect product of $\mathcal{L}(\mathcal{G})$ and the interval $[\mathcal{G}, \mathcal{C} \mathcal{S}$. A related mapping is studied in [8].

(C) 1984 Australian Mathematical Society $0263-6115 / 84 \$ A 2.00+0.00$ 
The first objective of this article is to consider two more embeddings of $\mathcal{L}(\mathcal{S})$ into certain subdirect products on the same pattern as explained above, with $\Re \mathscr{G}$ and $\mathcal{R} \mathcal{G}$, rectangular bands and rectangular groups, respectively, instead of $\mathcal{G}$. In [9], we have studied the variety $\mathcal{C}$ consisting of all central completely simple semigroups (those for which the product of pairs of idempotents lies in the centre of the containing maximal subgroup). The second objective of this paper is to show that $\mathfrak{L}(\mathcal{C S})$ is isomorphic to a subdirect product of $\mathcal{L}(\mathcal{C})$ and $[\mathcal{C}, \mathcal{C S}$ ] by means of a mapping analogous to the one mentioned above with groups. We have characterized in [9] the lattice $\mathcal{L}(\mathcal{C})$ of subvarieties of $\mathcal{C}$ in terms of the lattice $\mathfrak{L}(\mathcal{G})$. The variety $\mathcal{C}$ is thus the highest element of the lattice $\mathcal{E}(\mathcal{C S})$ for which the lattice of subvarieties is completely determined modulo group varieties. Hence at least the component $\mathcal{L}(\mathcal{C})$ of the subdirect decomposition of $\mathcal{L}(\mathcal{C S})$ mentioned above may be considered as of known structure. The congruences induced by these mappings are also explored and certain interesting consequences are deduced.

\section{Preliminaries}

In general, we use the notation and terminology of Howie [4] or Petrich [6]. In particular, we adopt the notation in [6] for Rees matrix semigroups, and use the description of congruences on a Rees matrix semigroup presented in [4]. In order to minimize the typographical complexity we modify the standard notation for a sandwich matrix and denote the $(j, k)$ th entry by $[j, k]$.

We will consistently use the following notation:

$\mathcal{G}=$ the variety of all groups,

$Q \mathcal{Q}=$ the variety of all abelian groups,

$\mathcal{T}=$ the variety of trivial groups,

$\Re \mathscr{B}=$ the variety of all rectangular bands,

$\Re \mathcal{G}=$ the variety of all rectangular groups (orthodox completely simple),

$\mathfrak{E}=$ the variety of all left zero semigroups,

$\Re=$ the variety of all right zero semigroups,

$\mathrm{CS}=$ the variety of all completely simple semigroups,

$[\mathcal{Q}, \mathscr{B}]=$ the interval of a lattice with minimum $\mathbb{Q}$ and maximum $\mathscr{B}$,

$\mathfrak{L}(\mathscr{V})=$ the variety of all subvarieties of a variety $\mathscr{V}$ of completely simple semigroups,

End $S=$ the semigroup of all endomorphisms of a semigroup $S$,

$\sigma_{X}=$ the semigroup of all transformations on a set $X$.

Let $N$ be a fully invariant subgroup of a free group on a countably infinite number of generators and denote by $थ$ the group variety corresponding to it.

$N(G)=$ the least fully invariant subgroup $H$ of the group $G$ for which $G / H \in$ थ. 
Lemma 2.1 [10]. Let $S=\mathfrak{T}(I, G, \Lambda ; P)$, where $P$ is normalized. Let $\varphi \in \mathscr{T}_{I}$, $\omega \in$ End $G, \psi \in \mathcal{T}_{\Lambda}$ be such that

$$
[\lambda, i] \omega=[1 \psi, 1 \varphi][\lambda \psi, 1 \varphi]^{-1}[\lambda \psi, i \varphi][1 \psi, i \varphi]^{-1} \quad(\lambda \in \Lambda, i \in I) .
$$

Then $\theta=\theta(\varphi, \omega, \psi)$ defined by

$$
(i, g, \lambda) \theta=\left(i \varphi,[1 \psi, i \varphi]^{-1}(g \omega)[1 \psi, 1 \varphi][\lambda \psi, 1 \varphi]^{-1}, \lambda \psi\right)
$$

is an endomorphism of $S$. Conversely, every endomorphism of $S$ can be so written uniquely.

A construction of the Rees matrix representation of a free completely simple semigroup follows.

LeMMA 2.2 ([2], [10]). Let $X=\left\{x_{i} \mid i \in I\right\}$ be a nonempty set, fix $1 \in I$ and let $I^{\prime}=I \backslash\{1\}$. Let

$$
Z=\left\{q_{i} \mid i \in I\right\} \cup\left\{[j, k] \mid j, k \in I^{\prime}\right\},
$$

$F_{Z}$ be the free group on $Z$, and let $P=([j, k])$ with $[1, k]=[j, 1]=1$, the identity of $F_{Z}$. Then

$$
F=\mathfrak{R}\left(I, F_{Z}, I ; P\right)
$$

is a free completely simple semigroup over $X$, with embedding $x_{i} \rightarrow\left(i, q_{i}, i\right)$,

Notation 2.3 . We fix a countably infinite set $X$, and in addition to the above notation, introduce

$$
F_{q}=\left\langle q_{i} \mid i \in I\right\rangle, \quad F_{p}=\left\langle[j, k] \mid j, k \in I^{\prime}\right\rangle,
$$

the free subgroups of $F_{Z}$ generated by the sets $\left\{q_{i} \mid i \in I\right\}$ and $\left\{[j, k] \mid j, k \in I^{\prime}\right\}$, respectively. We will consistently use the notation $F=\mathfrak{N}\left(I, F_{Z}, I ; P\right)$ introduced above.

We will need only fully invariant idempotent separating congruences, for they are precisely the ones which correspond to the varieties in the interval [ $\Re \mathscr{B}, \mathcal{C S}$ ]. In this context, the following special case of ([4], Lemma 4.19) is of particular interest.

LEMma 2.4. Let $S=\mathfrak{N}(I, G, \Lambda ; P)$. If $N$ is a normal subgroup of $G$, then $\rho_{N}$ defined on $S$ by

$$
(i, g, \lambda) \rho_{N}(j, h, \mu) \Leftrightarrow i=j, \quad g h^{-1} \in N, \lambda=\mu,
$$

is an idempotent separating congruence on $S$, and every such congruence is obtained in this way. Writing $P / N$ for the $\Lambda \times I$ matrix with the $(j, k)$ th entry equal to the $(j, k)$ th entry of $P$ modulo $N, S / \rho$ is isomorphic to $\mathfrak{R}(I, G / N, \Lambda ; P / N)$. 
Notation 2.5. We will consistently use the notation $\rho_{N}$ introduced above. For a variety $\mathcal{V}$ of completely simple semigroups, we denote by $\rho(\mathcal{V})$ the fully invariant congruence on $F$ corresponding to $\mathscr{V}$. Also let

$\tilde{E}\left(F_{Z}\right)=\left\{\omega \in\right.$ End $F_{Z} \mid$ there exist $\varphi, \psi \in \mathscr{T}_{I}$ such that (1) holds $\}$, $\mathcal{E}\left(F_{p}\right)=\left\{\omega \in\right.$ End $F_{p} \mid$ there exist $\varphi, \psi \in \mathscr{T}_{I}$ such that (1) holds $\}$.

Hence $\delta\left(F_{Z}\right)$ consists precisely of endomorphisms of $F_{Z}$ that arise in association with endomorphisms of $F$. The latter are uniquely determined by the functions $\left\{q_{i} \mid i \in I\right\} \rightarrow F_{Z}, \varphi, \psi \in \mathcal{T}_{I}$ independently. Furthermore, $\mathscr{E}\left(F_{p}\right)$ consists precisely of endomorphisms of $F_{p}$ that extend to elements of $\varepsilon\left(F_{Z}\right)$.

LEMMA 2.6 [10]. Let $N$ be a normal subgroup of $F_{Z}$. Then $\rho_{N}$ is fully invariant if and only if $N \omega \subseteq N$ for all $\omega \in \mathcal{E}\left(F_{Z}\right)$.

Definition 2.7. A normal subgroup of $F_{Z}$ (respectively, $F_{p}$ ) is $\varepsilon_{\text {-invariant if it }}$ is invariant under all $\omega \in \mathcal{E}\left(F_{Z}\right)$ (respectively, $\mathcal{E}\left(F_{p}\right)$ ). The set of all $\mathcal{E}$-invariant subgroups of $F_{Z}$ (respectively, $F_{p}$ ) will be denoted by $\Re$ (respectively, $\Re_{p}$ ). For any $N \in \mathfrak{T}$, let

$$
N_{q}=N \cap F_{q}, \quad N_{p}=N \cap F_{p}
$$

It is clear that $\mathscr{N}$ (respectively, $\mathfrak{N}_{p}$ ) is a sublattice of the lattice of all normal subgroups of $F_{Z}$ (respectively, $F_{p}$ ), and that each element of $\Re_{p}$ is the intersection with $F_{p}$ of an element of $\Re$ (for example, its normal closure in $F_{Z}$ ). It is also clear that $\mathscr{X}$ (respectively, $\mathfrak{X}_{p}$ ) contains every fully invariant subgroup of $F_{Z}$ (respectively, $F_{p}$ ).

Proposition 2.8 [10]. (i) The interval [R $\Re, \mathcal{C S}$ ] is anti-isomorphic to the lattice $\Re$. Consequently $[\Re \mathrm{R}, \mathrm{CS}]$ and $\mathcal{L}(\mathrm{CS})$ are modular lattices.

(ii) Moreover, $\mathscr{V} \in[\Re \mathscr{R}, \mathcal{C S}$ if and only if $\rho(\mathcal{V})$ is idempotent separating and so of the form $\rho_{N}, N \in \Re$. The mapping $\rho(\mathfrak{V}) \rightarrow N$, where $\rho(\mathbb{V})=\rho_{N}$, is an isomorphism of the lattice of fully invariant idempotent separating congrunences on $F$ onto $\Re$.

LEMma 2.9 [7]. If $N \in \mathfrak{R}$, then $N_{q}$ is a fully invariant subgroup of $F_{q}$ and $N_{q}\left(F_{Z}\right) \subseteq N$. If $\mathscr{V} \in \mathcal{L}(\mathcal{C S})$ is such that $\rho(\mathcal{V})=\rho_{N}$, then $\mathscr{V} \cap \mathcal{G}=\left\langle F_{q} / N_{q}\right\rangle$, the variety generated by $F_{q} / N_{q}$.

Notation 2.10. Let $\pi_{q}$ and $\pi_{p}$ be the projections of $F_{Z}$ onto $F_{q}$ and $F_{p}$, respectively. 
LEMMA 2.11 [9]. (i) $\pi_{q}, \pi_{p} \in \mathcal{E}\left(F_{Z}\right)$.

(ii) If $N \in \mathcal{X}$, then $N \pi_{q}=N_{q}, N \pi_{p}=N_{p}$.

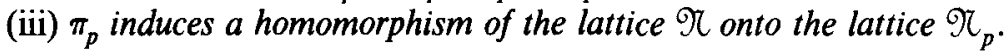

THEOREM 2.12 [7]. The mapping

$$
x: \mathcal{T} \rightarrow \mathscr{V} \cap \quad(\mathcal{V} \in \mathcal{L}(\mathcal{S}))
$$

is a homomorphism of $\mathcal{L}(\mathcal{C S})$ onto $\mathcal{L}(\mathcal{G})$. The congruence $\alpha$ induced by $\chi$ may be characterized on $[\Re \Re, \mathcal{C S}]$ as follows: for $\mathscr{Q}, \mathfrak{V} \in\left[\Re \mathscr{R}, \mathcal{C}\right.$ ] with $\rho(\mathcal{Q})=\rho_{M}$, $\rho(\mathfrak{V})=\rho_{N}$

$$
\mathscr{Q} \alpha \mathscr{V} \Leftrightarrow M_{q}=N_{q} .
$$

In particular, $(\mathcal{Q}, \mathcal{G}) \in \alpha$ if and only if $M_{q}=\{1\}$.

Moreover, the mapping

$$
\mathfrak{V} \rightarrow(\mathfrak{V} \cap \mathcal{G}, \mathcal{V} \vee \mathcal{G}) \quad(\mathcal{V} \in \mathcal{L}(\mathcal{C S}))
$$

is an isomorphism of $\mathfrak{L}(\mathcal{C S})$ into $\mathfrak{L}(\mathcal{G}) \times[\mathcal{G}, \mathcal{C S}]$.

Notation 2.13 [7]. For any $\mathcal{V} \in \mathcal{L}(\mathcal{C} S)$, let

$$
\overline{\mathcal{V}}=\{S \in \mathcal{C} \mid \text { all subgroups of } S \text { are in } \mathbb{V}\} \text {. }
$$

It is easily verified that $\overline{\widetilde{V}}$ is a variety.

Proposition 2.14 [7]. For any $\mathfrak{V} \in \mathcal{L}(\mathcal{C S})$, with $\rho(\mathfrak{V})=\rho_{N}, N \in \mathfrak{N}$, we have $\rho(\overline{\mathscr{V}})=\rho_{N_{q}\left(F_{Z}\right)}$.

A certain variety considered in [9] will figure prominently here as well.

Definition 2.15. A completely simple semigroup $S$ is central if the product of any two idempotents of $S$ lies in the centre of the containing maximal subgroup. The class of all central completely simple semigroups will be denoted by $\mathcal{C}$.

Notation 2.16. For any subgroups $H$ and $K$ of a group $G$, we denote by [ $H, K]$ the subgroup of $G$ generated by the elements of the form $h^{-1} k^{-1} h k$ where $h \in H, k \in K$. In particular, we write $G^{\prime}=[G, G]$ for the commutator subgroup of $G$. For any $a, b \in G, a^{b}=b^{-1} a b$. Furthermore, for any subgroup $H$ of $F_{Z}$, we will write $H^{\wedge}$ for the normal closure of $H$ in $F_{Z}$.

Proposition 2.17 ([8] or Example 4.13 in [5]). The class $\mathcal{C}$ is the variety of completely simple semigroups for which $\rho(\mathcal{C})=\rho_{K}$, where $K=\left[F_{Z}, F_{p} \hat{\jmath}\right.$. 
The following observations from lattice theory regarding neutral elements will be useful. The results cited here can be found in Birkhoff [1].

Notation 2.18. For any element $a$ in a lattice $L$, let

$$
(a]=\{x \in L \mid x \leqslant a\}, \quad[a)=\{x \in L \mid x \geqslant a\} .
$$

Definition 2.19. An element $a$ in a lattice $L$ is said to be neutral if, for all elements $x, y$ in $L$, the sublattice generated by $a, x$ and $y$ is distributive.

LEMMA 2.20 [1]. The neutral elements in a lattice form a distributive sublattice.

LEMMA 2.21 [1]. If $a$ is a neutral element in a lattice $L$, then

(i) the mappings $x \rightarrow x \vee a$ and $x \rightarrow x \wedge a(x \in L)$ are both endomorphisms of $L$,

(ii) the mapping $x \rightarrow(x \wedge a, x \vee a)$ is a monomorphism of $L$ into $(a] \times[a)$.

LEMMA 2.22 [1]. Let $L$ be a modular lattice. Then $a \in L$ is neutral if either of the mappings

$$
x \rightarrow x \wedge a, \quad x \rightarrow x \vee a \quad(x \in L),
$$

is an endomorphism.

\section{An embedding related to rectangular bands}

Although the principal concern of this paper is the variety $\mathcal{C}$, for the sake of completeness, we consider briefly in this and the following section two simpler cases.

The principal result considered here is the embedding of the variety $\mathcal{S} S$ of completely simple semigroups by means of the mapping $\delta \rightarrow(\delta \cap \Re \mathscr{B}, S \vee \Re \mathscr{B})$ onto a subdirect product of $\mathcal{L}(\Re \mathscr{R})$ and $[\Re \mathscr{R}, \mathcal{C}$ ] to be specified exactly.

LEMMA 3.1 [9]. The mapping $\chi_{1}: \mathfrak{V} \rightarrow \mathfrak{V} \cap \mathscr{R} \mathscr{B}(\mathfrak{V} \in \mathcal{L}(\mathcal{C S}))$ is a homomorphism of $\mathcal{L}(\mathcal{C S})$ onto $\mathcal{L}(\mathscr{R} \mathscr{B})$.

COROLlaRY 3.2. The mapping $\chi_{2}: \mathfrak{V} \rightarrow \mathfrak{V} \vee \Re \Re(\mathcal{V} \in \mathcal{L}(\mathcal{C S})$ ) is a homomorphism of $\mathcal{E}(\mathcal{C S})$ onto $[\Re \mathscr{R}, \mathcal{C S}]$.

Proof. By Proposition 2.8, $\mathcal{L}(\mathcal{C S})$ is a modular lattice so that it follows from Lemmas 3.1, 2.22 and 2.21 that $\chi_{2}$ is a homomorphism of $\mathcal{L}(\mathcal{S})$ onto [ $\Re, \mathcal{C}$ ]. 
THEOREM 3.3. The mapping $\chi: \mathfrak{V} \rightarrow(\mathfrak{V} \cap \Re \mathscr{B}, \mathfrak{V} \vee \Re \mathscr{B})(\mathfrak{V} \in \mathcal{L}(\mathcal{C S}))$ is an isomorphism of $\mathcal{L}(\mathrm{CS})$ onto the subdirect product

$$
\{(\mathscr{Q}, \mathscr{W}) \in \mathcal{L}(\Re \mathscr{B}) \times[\Re \mathscr{B}, \mathcal{C S}] \mid \mathcal{Q} \neq \mathfrak{R} \mathscr{B} \Rightarrow \mathscr{W} \in \mathcal{L}(\mathfrak{R S})\} .
$$

Proof. That $\chi$ is a monomorphism is a special case of Proposition 3.4 of Hall and Jones [3]. Alternatively, it is a consequence of Lemmas 2.21 and 3.1. It remains to characterize the image.

If $\mathscr{V} \in \mathcal{L}(\mathcal{S})$ is such that $\mathscr{U}=\mathscr{V} \cap \Re \mathscr{B} \neq \mathscr{R} \mathscr{B}$, then $\mathscr{W}=\mathscr{V} \vee \mathscr{R} \in$ $\mathcal{L}(\Re \mathcal{G})$. Conversely, let $(\mathscr{W}, \mathscr{W}) \in \mathcal{L}(\Re \mathscr{B}) \times[\Re \mathscr{B}, \mathcal{C}$ ]. If $\mathscr{U} \neq \Re \mathscr{B}$ and $\mathscr{W}=$

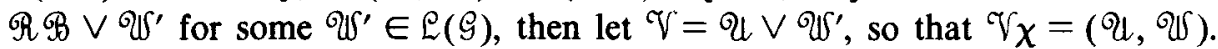
We also have $\mathfrak{V} \chi=(\Re \mathscr{G}, \mathscr{V})$ for any $\mathscr{V} \in[\Re \mathscr{R}, \mathcal{C}]$ ]. This shows that $\chi$ maps $\mathfrak{L}(\mathcal{S})$ onto the set in the statement of the theorem, which is evidently a subdirect product of $\mathcal{L}(\mathcal{R} \mathscr{B})$ and $[\Re \mathscr{B}, \mathcal{C S}$.

It is somewhat instructive to know what the congruences are which are induced by the homomorphisms $\chi_{1}$ and $\chi_{2}$.

Proposition 3.4. The classes of the congruence induced by $\chi_{1}$ in Lemma 3.1 are

$$
\mathfrak{L}(\mathcal{S}),[\mathfrak{L}, \mathfrak{L} \vee \mathcal{G}],[\Re, \Re \vee \mathcal{S}],[\Re \mathscr{R}, \mathcal{C S}] \text {. }
$$

The classes of the congruence induced by $\chi_{2}$ in Corollary 3.2 are

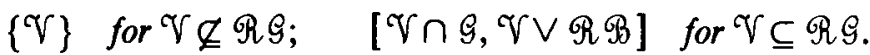

Proof. The assertion concerning $\chi_{1}$ is checked readily. We now prove the contention concerning $\chi_{2}$.

Let $\mathscr{V} \nsubseteq \Re \mathcal{G}$ and $\mathcal{Q} \vee \Re \mathscr{B}=\mathscr{V} \vee \Re \mathscr{B}$. Then $\mathcal{Q} \not \Re \mathcal{G}$ and thus $\Re \mathscr{B} \subseteq \mathcal{Q} \cap \mathcal{V}$ whence $\mathcal{Q}=\mathscr{T}$.

Next let $\mathscr{V} \subseteq \Re \mathcal{G}$. If $\mathcal{Q} \vee \Re \mathscr{B}=\mathscr{V} \vee \Re \mathscr{B}$, then by the above, $\mathscr{Q} \subseteq R \mathcal{G}$ so that $\mathcal{Q} \cap \mathcal{G}=\mathscr{V} \cap \mathcal{G}$, whence $\mathcal{Q} \in[\mathcal{T} \cap \mathcal{G}, \mathcal{V} \vee \Re \mathscr{B}]$. Conversely, assume that $\mathcal{U} \in$ $[\mathfrak{V} \cap \mathcal{S}, \mathfrak{V} \vee \Re \mathscr{B}$ ]. Then $\mathscr{V} \cap \mathcal{S} \subseteq \mathcal{Q} \subseteq \mathfrak{V} \vee \Re \mathscr{B} \subseteq \Re \mathcal{G}$. Intersecting with $\mathcal{G}$, we deduce that $\mathscr{U} \cap \mathcal{S}=\mathscr{V} \cap \mathcal{S}$, and thus

$$
\mathscr{U} \vee \mathfrak{R}=(\mathscr{U} \cap \mathcal{G}) \vee \mathfrak{R}=(\mathscr{T} \cap \mathcal{G}) \vee \mathscr{R} \mathscr{B}=\mathscr{T} \vee \mathfrak{R},
$$

as required.

\section{An embedding related to rectangular groups}

The result here is analogous to that in the preceding section; this time the role of rectangular bands is played by rectangular groups. For the next result recall Notation 2.13 for $\overline{\text { Q }}$. 
THEOREM 4.1. The mapping $\tau: \mathcal{V} \rightarrow(\mathfrak{V} \cap \Re \mathcal{R}, \mathcal{V} \vee \Re \mathcal{G})(\mathfrak{V} \in \mathcal{L}(\mathcal{S}))$ is an isomorphism of $\mathcal{L}(\mathcal{C S})$ onto the subdirect product

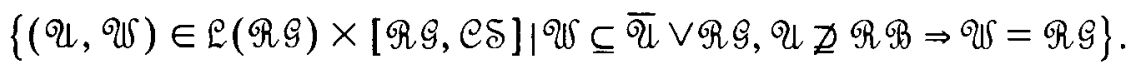

Proof. By Theorems 2.12 and 3.3, the varieties $\mathcal{G}$ and $\Re \mathscr{B}$ are neutral elements in the lattice $\mathcal{L}(\mathcal{C S})$ so that, by Lemma $2.20, \Re \mathcal{G}=\mathcal{G} \vee \Re \mathscr{B}$ is also a neutral element in $\mathcal{L}(\mathcal{S S})$. It follows that $\tau$ is a monomorphism of $\mathcal{L}(\mathcal{S S})$ onto a subdirect product of $\mathcal{L}(\Re \mathcal{G})$ and $[\Re \mathcal{G}, \mathcal{S}]$. It only remains to characterize the image.

Let $\mathscr{V} \in \mathcal{L}(\mathcal{S S})$. Then letting $\mathscr{U}=\mathscr{V} \cap \Re \mathcal{G}$ and $\mathscr{W}=\mathfrak{V} \vee \Re \mathcal{G}$, we get

$$
\begin{aligned}
& \mathscr{W}=\mathscr{V} \vee \Re \mathcal{R} \subseteq \overline{(\mathfrak{V} \cap \mathcal{G})} \vee \Re \mathcal{G}=\overline{(\mathcal{V} \cap \Re \mathcal{G})} \vee \Re \mathcal{G} \\
& =\overline{\mathcal{Q}} \vee \Re \mathcal{G}
\end{aligned}
$$

if also $\mathscr{Q} \Re \mathscr{B}$, then $\mathscr{V}$ is contained in $\Re \mathcal{G}$ and thus $\mathscr{W}=\mathscr{V} \vee \Re \mathcal{G}=\Re \mathcal{G}$. If we denote by $\mathscr{P}$ the last set in the statement of the theorem, we have proved that $\tau$ maps $\mathcal{L}(\mathcal{C S})$ into $\mathscr{P}$. Conversely, let $(\mathcal{Q}, \mathcal{W}) \in \mathscr{P}$. If $\mathcal{Q} \nsupseteq \mathfrak{R} \mathscr{B}$, then let $\mathscr{V}=\mathscr{Q}$ so that $\mathcal{V}_{\tau}=(\mathcal{Q}, \mathscr{R} \mathcal{G})$. Assume that $\mathscr{Q} \supseteq \Re \mathscr{B}$ and let $\mathcal{V}=\overline{\mathcal{Q}} \cap \mathcal{W}$. Then

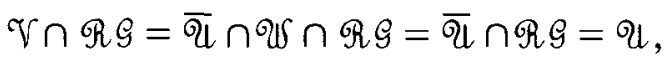

$$
\begin{aligned}
& \widetilde{ } \vee \Re \mathcal{G}=(\overline{\mathcal{U}} \cap \mathscr{W}) \vee \Re \mathcal{G}=(\overline{\mathcal{Q}} \vee \Re \mathcal{G}) \cap \mathscr{W}=\mathscr{W} \text {, }
\end{aligned}
$$

where the last but one equality follows from the fact that $\mathscr{R S}$ is neutral in $\mathscr{L}(\mathcal{S})$. Consequently, $\mathcal{V}_{\tau}=(\mathscr{\vartheta}, \mathscr{\mho})$ as required.

Recall the following notation from [7]; for $\mathcal{Q}, \mathcal{V} \in \mathcal{E}(\mathcal{C S})$,

$$
\text { U } \boldsymbol{\beta} \mathfrak{V} \Leftrightarrow \mathscr{U} \vee \mathcal{G}=\mathfrak{T} \vee \mathcal{G}
$$

From Theorem 4.1, it follows that the mappings

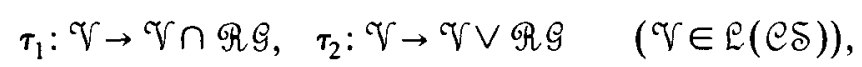

are retractions of $\mathcal{L}(\mathcal{C S})$.

The classes of the congrunces induced by $\tau_{1}$ and $\tau_{2}$ can be described as follows.

Proposition 4.2. The classes of the congruence induced by $\tau_{1}$ are

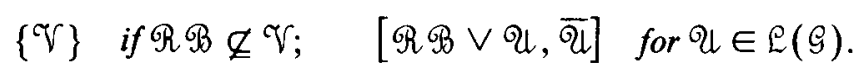

The classes of the congruence induced by $\tau_{2}$ are

$$
\mathcal{L}(\Re \mathcal{G}) ; \quad \beta \text {-class } \cap(\mathfrak{L}(\mathcal{C S}) \backslash \mathfrak{L}(\Re \mathcal{G})) .
$$


PRoof. Let $\mathcal{Q}, \mathfrak{V} \in \mathcal{E}(\mathcal{C S})$. First note that $\mathfrak{V} \cap \mathcal{R}=(\mathfrak{V} \cap \Re \mathscr{B}) \vee(\mathfrak{V} \cap \mathcal{G})$ which implies

$$
\begin{aligned}
& \mathscr{U} \cap \Re \mathcal{G}=\mathscr{V} \cap \mathfrak{R} \Leftrightarrow(\mathcal{U} \cap \Re \mathscr{B}) \vee(\mathscr{U} \cap \mathcal{G})=(\mathfrak{V} \cap \Re \mathscr{B}) \vee(\mathscr{V} \cap \mathcal{G}) \\
& \Leftrightarrow \mathscr{Q} \cap \Re \mathscr{B}=\mathscr{V} \cap \Re \mathscr{B}, \quad \mathscr{Q} \cap \mathcal{G}=\mathfrak{V} \cap \mathcal{G} .
\end{aligned}
$$

The assertion concerning $\tau_{1}$ follows from this and the definition of $\overline{\mathcal{Q}}$.

If $\mathscr{Q} \vee \Re \mathcal{G}=\mathscr{} \vee \Re \mathcal{G}$ and $\mathscr{V} \subseteq \Re \mathcal{G}$, then $\mathscr{Q} \vee \Re \mathcal{G}=\mathscr{V} \vee \Re \mathcal{G}=\Re \mathcal{G}$ and thus $\mathscr{Q} \subseteq \Re \mathcal{R}$; conversely, if $\mathscr{Q}, \mathcal{V} \subseteq \Re \mathcal{G}$, then trivially $\mathcal{Q} \vee \Re \mathcal{G}=\mathscr{V} \vee \Re \mathcal{G}$.

Now assume that $\mathscr{Q} \vee \Re \mathcal{G}=\mathscr{T} \vee \Re \mathcal{G}$ and $\mathscr{V} \notin \Re \mathcal{G}$. Then $\Re \mathcal{G} \subset \mathcal{V} \vee \Re \mathcal{G}$ and thus $\mathscr{R} \mathscr{B} \subseteq \mathcal{Q} \cap \mathcal{T}$, so that

$$
\mathscr{Q} \vee \mathcal{G}=(\mathscr{Q} \vee \Re \mathscr{B}) \vee \mathcal{G}=\mathscr{U} \vee \mathfrak{R}=\mathscr{V} \vee \mathfrak{R}=\mathfrak{T} \vee \mathcal{G}
$$

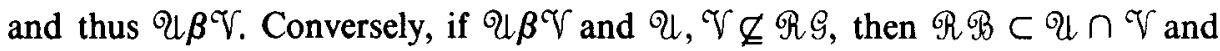
thus $\mathscr{Q} \vee \Re \mathcal{G}=\mathscr{Q} \vee \mathcal{G}=\mathscr{V} \vee \mathcal{G}=\mathscr{V} \vee \mathfrak{R} \mathcal{G}$, as required.

\section{Certain subgroups of a free group}

The results proved in this section will be used in the next section where we consider an embedding of $\mathcal{L}(\mathcal{C S})$ into $\mathcal{L}(\mathcal{C}) \times[\mathcal{C}, \mathcal{S}]$ of the general form discussed in the preceding two sections. They concern subgroups of a free group closed under, what we call, almost complete deletion, and seem to be of intrinsic interest.

Notation 5.1. Let $G$ be a free group on a nonempty set $X$. We will denote by $\mathcal{S}$ the set of all normal subgroups $H$ of $G$ which are closed under almost complete deletion, that is,

$$
u\left(x_{1}, x_{2}, \ldots, x_{n}\right) \in H \Rightarrow u(1,1, \ldots, 1, x, 1, \ldots, 1) \in H,
$$

where $x$ is any element of $X$ in the $i$ th position for any $i=1,2, \ldots, n$.

Condition (2) is equivalent to requiring that $H$ be closed under all endomorphisms $\theta$ of $G$ defined on the generators $X$ as follows: let $x, z \in X$; then for $y \in X$

$$
y \theta= \begin{cases}x & \text { if } y=z \\ 1 & \text { otherwise. }\end{cases}
$$

Observe that $S$ is a sublattice of the lattice of normal subgroups of $G$ containing all fully invariant subgroups of $G$.

Proposition 5.2. The mapping $\theta: H \rightarrow H G^{\prime}(H \in \mathcal{S})$ is a retraction of $\mathcal{S}$ onto the sublattice $\mathfrak{Q}$ of fully invariant subgroups containing $G^{\prime}$. 
Proof. Let $H \in \mathfrak{s}$. It is clear that $H \theta=H G^{\prime} \in \mathcal{S}$ and in order to show that $H \theta \in \mathbb{Q}$, we wish to establish that $H \theta$ is a fully invariant subgroup of $G$. We do this in the following lemma.

LEMMA 5.3. For $H \in \mathfrak{S}, H G^{\prime}$ is a fully invariant subgroup of $G$.

Proof. Let $a \in H G^{\prime}$. Then $a$ can be written in the form

$$
a=x_{1}^{r_{1}} \cdots x_{k}^{r_{k}} g
$$

where $x_{i} \in X, g \in G^{\prime}, r_{i} \neq 0$ and $x_{i} \neq x_{j}$ if $i \neq j$. Let $r$ be the smallest positive exponent $r_{i}$ appearing in an expression of the form (3) for some $a \in H G^{\prime}$. Without loss of generality $r=r_{1}$. By (2) we then have

$$
a(x, 1, \ldots, 1)=x^{r} \in H G^{\prime},
$$

for all $x \in X$. Hence, by the minimality of $r$, for any element of the form (3) in $H G^{\prime}$, there must exist $s_{i}$ such that $r_{i}=r s_{i}$ and $a$ can be written in the form

$$
a=x_{1}^{r s_{1}} \cdots x_{k}^{r s_{k}} g \text {. }
$$

Conversely, any element of the form (5) must lie in $H G^{\prime}$ by (4). Thus $H G^{\prime}$ consists precisely of those elements of $G$ that can be written in the form (5) and is therefore fully invariant.

We now go back to the proof of Proposition 5.2.

Clearly $\theta$ is a join preserving mapping. For $H, K \in \delta$ we wish to establish that

$$
H G^{\prime} \cap K G^{\prime}=(H \cap K) G^{\prime} .
$$

Let $g$ be an element in the left-hand side of this equation. Then $g$ can be written in the form

$$
g=g\left(x_{1}, \ldots, x_{l}\right)=x_{1}^{r_{1}} \cdots x_{k}^{r_{k}} c,
$$

where $c \in G^{\prime}$. Now if $c=c\left(x_{1}, \ldots, x_{l}\right)$, then since $c$ is a product of conjugates of commutators and any commutator in a single variable is the identity, we have, with $x_{j}$ replacing $x_{i}$,

$$
c\left(1, \ldots, 1, x_{j}, 1, \ldots, 1\right)=1
$$

for all $i$. Hence

$$
\begin{aligned}
x_{j}^{r_{i}} & =x_{j}^{r_{i}} c\left(1, \ldots, 1, x_{j}, 1, \ldots, 1\right) \\
& =g\left(1, \ldots, 1, x_{j}, 1, \ldots, 1\right) \in H G^{\prime} \cap K G^{\prime},
\end{aligned}
$$

for all $i, j$. Let $r=$ g.c.d. $\left(r_{i}\right)$, then we must have $x_{i}^{r} \in H G^{\prime} \cap K G^{\prime}$, for all $i$. Thus, for some $h \in H, k \in K, a, b \in G^{\prime}$,

$$
x_{i}^{r}=h a=k b .
$$


Let $h=h\left(x_{1}, \ldots, x_{m}\right), a=a\left(x_{1}, \ldots, x_{m}\right)$, where $i \leqslant m$. Then $a\left(1, \ldots, 1, x_{i}, 1, \ldots, 1\right)=1$, since $a \in G^{\prime}$, and so

$$
\begin{aligned}
x_{i}^{r} & =h\left(1, \ldots, 1, x_{i}, 1, \ldots, 1\right) a\left(1, \ldots, 1, x_{i}, 1, \ldots, 1\right) \\
& =h\left(1, \ldots, 1, x_{i}, 1, \ldots, 1\right) \in H,
\end{aligned}
$$

for all $i$. Therefore, $x_{1}^{r_{1}} \cdots x_{k}^{r_{k}} \in H$. Similarly, $x_{1}^{r_{1}} \cdots x_{k}^{r_{k}} \in H \cap K$ and $g \in$ $(H \cap K) G^{\prime}$. Thus $H G^{\prime} \cap K G^{\prime} \subseteq(H \cap K) G^{\prime}$, while the reverse containment is trivial. Hence $\theta$ preserves meets and is a lattice homomorphism. Since $\theta$ clearly maps elements of $\mathbb{Q}$ identically, the proof is complete.

Proposition 5.2 suffices for our main purposes in the next section. In addition it has the following interesting consequence.

COROLlary 5.4. The derived subgroup $G^{\prime}$ is a neutral element in the modular lattice $\mathcal{S}$.

Proof. Since $\delta$ is a sublattice of the lattice of normal subgroups of $G$, it follows that $\delta$ is modular. From Proposition 5.2 and Lemma 2.22, it follows that $G^{\prime}$ is a neutral element in $\delta$.

\section{An embedding related to $C$}

The principal result here is analogous to the principal results in Sections 3 and 4 , and it now concerns a subdirect product related to the variety $\mathcal{C}$ of all central completely regular semigroups. We shall see, however, that the proof for this case is considerably more complex than those in Sections 3 and 4.

Lemma 6.1. For $N \in \mathfrak{T}, N_{p}$ is closed under almost complete deletion when considered as a subgroup of the free group $F_{p}$ on the set of free generators $\left\{[j, k] \mid j, k \in I^{\prime}\right\}$.

Proof. Let $j_{0}, j_{1}, k_{0}, k_{1} \in I^{\prime}$. It suffices to establish that there exists $\omega \in \mathcal{E}\left(F_{p}\right)$ such that for $j, k \in I^{\prime}$,

$$
[j, k] \omega= \begin{cases}{\left[j_{1}, k_{1}\right]} & \text { if }[j, k]=\left[j_{0}, k_{0}\right], \\ 1 & \text { otherwise. }\end{cases}
$$

Let $\varphi, \psi \in \mathcal{T}_{I}$ be defined by

$$
k \varphi=\left\{\begin{array}{ll}
k_{1} & \text { if } k=k_{0}, \\
1 & \text { otherwise, }
\end{array} \quad j \psi= \begin{cases}j_{1} & \text { if } j=j_{0}, \\
1 & \text { otherwise. }\end{cases}\right.
$$


Now define $\omega$ to be the endomoprhism of $F_{p}$ given by the action on the free generators $\left\{[j, k] \mid j, k \in I^{\prime}\right\}$, defined by equation (1) in Lemma 2.1.

By its very definition $\omega \in \mathcal{E}\left(F_{p}\right)$ and clearly $\omega$ satisfies (6) above.

Lemma 6.2. Let $n \in N \in \mathfrak{N}$. Then there exist $a \in N_{q}, g \in N \cap\left[F_{q}, F_{p} \hat{]}\right.$ and $b \in N_{p}$ such that

$$
n=a g b
$$

Consequently,

$$
N=N_{q}\left(N \cap\left[F_{q}, F_{p}\right]^{\wedge}\right) N_{p}=N_{q}\left(N \cap\left[F_{Z}, F_{p}\right]^{\wedge}\right) N_{p} .
$$

Proof. We have $n=a_{1} b_{1} a_{2} b_{2} \cdots a_{k} b_{k}$, for some $a_{i} \in F_{q}, b_{i} \in F_{p}$. Hence

$$
\begin{aligned}
n & =a_{1} b_{1}\left(a_{2} b_{1}^{-1} a_{2}^{-1} b_{1}\right) b_{1}^{-1} a_{2} b_{1} b_{2} a_{3} \cdots \\
& =a_{1}\left[a_{2}^{-1}, b_{1}\right]^{b_{1}^{-1}} a_{2} b_{1} b_{2} a_{3} \cdots \\
& =a_{1} a_{2}\left(\left[a_{2}^{-1}, b_{1}\right]^{b_{1}^{-1}}\right)^{a_{2}} b_{1} b_{2} a_{3} \cdots \\
& =\cdots \\
& =a_{1} a_{2} \cdots a_{k} g b_{1} b_{2} \cdots b_{k}
\end{aligned}
$$

where $g$ is a product of conjugates of elements of the form $\left[a_{2}^{-1}, b_{1}\right],\left[a_{3}^{-1}, b_{1} b_{2}\right] \cdots$. These commutators all lie in $\left[F_{q}, F_{p}\right]$. Hence $g$ lies in $\left[F_{q}, F_{p}\right]$. Now

$$
a_{1} a_{2} \cdots a_{k}=n \pi \in N_{q}
$$

and

$$
b_{1} b_{2} \cdots b_{k}=n \pi_{p} \in N_{p} .
$$

Therefore, from (9) we deduce that $g \in N$ and so $g \in N \cap\left[F_{q}, F_{p} \hat{]}\right.$. This establishes (7) and so also, by (10) and (11), that $N \subseteq N_{q}\left(N \cap\left[F_{q}, F_{p}\right]\right) N_{p}$. Since the reverse containment clearly holds, the first equality in (8) is also verified. The second equality follows easily.

Proposition 6.3. The mapping

$$
\nu: N \rightarrow N\left[F_{Z}, F_{p}\right]^{\wedge} \quad(N \in \Re),
$$

is a retraction of $\mathcal{X}$ onto the interval $\left[\left[F_{Z}, F_{p} \hat{]}, F_{Z}\right]\right.$.

Proof. It is clear that $\nu$ maps $\mathcal{N}$ onto the stated interval and maps elements of the interval identically. It is also clear that $\nu$ respects the join operation. It remains to show that $\nu$ respects the meet operation. So for $M, N \in \mathscr{N}$, we wish to establish the equality

$$
(M \cap N)\left[F_{Z}, F_{p}\right]^{\wedge}=M\left[F_{Z}, F_{p}\right]^{\wedge} \cap N\left[F_{Z}, F_{p}\right]^{\hat{n}} .
$$


Since the left side of (12) is clearly contained in the right, we consider any element $u$ in the right side. Then there exist $m \in M, n \in N$ and $r, s \in\left[F_{Z}, F_{p}\right]$ such that

$$
u=m r=n s .
$$

By Lemma 6.2, there exist $a \in M_{q}, c \in N_{q}, b \in M_{p}, d \in N_{p}, g \in M \cap\left[F_{q}, F_{p} \hat{]}\right.$ and $h \in N \cap\left[F_{q}, F_{p}\right]$ such that

$$
m=a g b, \quad n=c h d .
$$

Applying $\pi_{q}$ to (13), we obtain

$$
a=(m r) \pi_{q}=(n s) \pi_{q}=c \in M_{q} \cap N_{q} \subseteq M \cap N,
$$

so that $g b r=h d s$. Applying $\pi_{p}$, we obtain $b\left(r \pi_{p}\right)=d\left(s \pi_{p}\right)$ where $r \pi_{p}, s \pi_{p} \in F_{p}^{\prime}$, $b \in M_{p}, d \in N_{p}$. By Lemma 6.1 and Proposition 5.2, we have

$$
M_{p} F_{p}^{\prime} \cap N_{p} F_{p}^{\prime}=\left(M_{p} \cap N_{p}\right) F_{p}^{\prime}
$$

and, since $b\left(r \pi_{p}\right)=d\left(s \pi_{p}\right) \in M_{p} F_{p}^{\prime} \cap N_{p} F_{p}^{\prime}$, there exist $v \in M_{p} \cap N_{p}, w \in F_{p}^{\prime}$ with $b\left(r \pi_{p}\right)=d\left(s \pi_{p}\right)=v w$. Hence

$$
\begin{aligned}
u & =a g b r=a b g^{b} r \\
& =a b\left(r \pi_{p}\right)\left(r \pi_{p}\right)^{-1} g^{b} r=a v w\left(r \pi_{p}\right)^{-1} g^{b} r \\
& \in(M \cap N)\left(M_{p} \cap N_{p}\right) F_{p}^{\prime} F_{p}^{\prime}\left[F_{q}, F_{p}\right]^{\wedge}\left[F_{Z}, F_{p}\right]^{-} \\
& \subseteq(M \cap N)\left[F_{Z}, F_{p}\right],
\end{aligned}
$$

so that the required containment in (12) holds, completing the proof of the proposition.

COROllary 6.4. $\left[F_{Z}, F_{p} \hat{]}\right.$ is a neutral element in $\mathfrak{T}$.

Proof. Since $\mathfrak{T}$ is modular, the result follows from Proposition 6.3 and Lemma 2.22 .

COROLLARY 6.5. The mapping $\eta_{1}: \mathcal{V} \rightarrow \mathfrak{V} \cap \mathcal{C}(\mathcal{V} \in \mathcal{L}(\mathcal{C S})$ ), is a retraction of $\mathcal{E}(\mathcal{C S})$ onto $\mathcal{L}(\mathcal{C})$.

Proof. In view of Corollary 6.4 , in order to verify that $\eta_{1}$ is a homomorphism, it suffices to consider the case $\mathcal{Q}, \mathcal{V} \in \mathcal{L}(\mathcal{S}), \mathcal{Q} \supseteq \Re \mathscr{R}, \mathscr{\Upsilon} \nsupseteq \Re$, for join. Let 
$\mathcal{V}^{\prime}=\Re \mathscr{R} \vee \mathcal{V}$; we note that $\mathfrak{V} \subseteq \mathcal{C}$ and $R \mathscr{B} \subseteq \mathcal{V}^{\prime} \subseteq \mathcal{C}$. Now using Corollary 6.4, we obtain

$$
\begin{aligned}
& \mathcal{Q} \eta_{1} \vee \mathscr{V} \eta_{1}=(\mathscr{U} \cap \mathcal{C}) \vee(\mathscr{C} \cap)=[(\mathscr{Q} \cap \mathcal{C}) \vee \mathscr{R}] \vee \mathfrak{W} \\
& =(\mathscr{U} \cap \mathcal{C}) \vee \mathcal{V}^{\prime}=(\mathscr{Q} \cap \mathcal{C}) \vee\left(\mathscr{V}^{\prime} \cap \mathcal{C}\right) \\
& =\left(\mathscr{U} \vee \mathfrak{V}^{\prime}\right) \cap \mathcal{C}=(\mathscr{U} \vee \Re \mathscr{B} \vee \mathfrak{V}) \cap \mathcal{C} \\
& =(\mathscr{U} \vee \mathfrak{V}) \cap \mathcal{e}=(\mathscr{U} \vee \mathfrak{V}) \eta_{1}
\end{aligned}
$$

as required.

Corollary 6.6. The mapping $\eta: \mathcal{T} \rightarrow(\mathfrak{V} \cap \mathcal{C}, \mathfrak{V} \vee \mathcal{C})(\mathfrak{V} \in \mathcal{E}(\mathcal{C S}))$, is an isomorphism of $\mathrm{L}(\mathrm{CS})$ onto a subdirect product of $\mathrm{C}(\mathrm{C})$ and $[\mathrm{C}, \mathrm{eS}$, that is, $\mathrm{e}$ is a neutral element in $\mathcal{L}(\mathrm{CS})$.

Proof. This follows directly from Corollary 6.5, Proposition 2.8, Lemma 2.21 and Lemma 2.22 .

\section{The image of $\mathcal{L}(\mathcal{S})$ under $\eta$}

In order to precisely locate the image of $\mathscr{E}(\mathcal{S})$ under $\eta$ in the direct product $\mathcal{L}(\mathcal{C}) \times[\mathcal{C}, \mathcal{C}]$, see Corollary 6.6 , we need some preparation. This discussion also provides further insight into the various subgroups of the free group $F_{Z}$ as well as of the associated varieties.

Definition 7.1. Let $N \in \Re_{p}$. The depth of $N$, denoted by $d(N)$, is the smallest positive integer $d$ such that $[j, k]^{d} \in N$, for all $j, k \in I^{\prime}$, if such an integer exists, otherwise $d(N)=\infty$. If $\mathscr{V} \in \mathcal{L}(\mathrm{e} S)$ is such that $\rho(\mathcal{V})=\rho_{N}$, then the depth of $\mathscr{V}$ or of $N$ is the depth of $N_{p}$ and we write $d(\mathscr{V})=d(N)=d\left(N_{p}\right)$. If $\mathscr{V} \in \mathcal{L}(\mathcal{S})$ and $\mathscr{R} \mathscr{G} \mathfrak{V}$, then the depth of $\mathfrak{V}$ is 1 .

Lemma 7.2. Let $N \in \Re$. If $\left[j^{\prime}, k^{\prime}\right]^{d} \in N$ for some $j^{\prime}, k^{\prime} \in I^{\prime}$, then $[j, k]^{d} \in N$, for all $j, k \in I^{\prime}$.

Proof. Let $j, k \in I^{\prime}$. Define $\varphi, \psi$ on $I$ by

$$
r \psi=\left\{\begin{array}{ll}
j & \text { if } r=j^{\prime}, \\
1 & \text { otherwise, }
\end{array} \quad r \varphi= \begin{cases}k & \text { if } r=k^{\prime}, \\
1 & \text { otherwise, }\end{cases}\right.
$$

and let $\omega \in \mathcal{E}\left(F_{p}\right)$ be defined by equation (1) in Lemma 2.1. Then $\left[j^{\prime}, k^{\prime}\right] \omega=$ $[j, k]$ so that $\left[j^{\prime}, k^{\prime}\right]^{d} \in N$ implies that $[j, k]^{d} \in N$, as required. 
As an immediate consequence, we have

COROLLARY 7.3. Let $N \in \mathfrak{R}$ and let $j, k \in I^{\prime}$. If $d$ is the smallest positive integer such that $[j, k]^{d} \in N$, then $d=d(N)$.

Notation 7.4. For any positive integer $d$, let $K_{d}$ be the smallest element of $\Re_{p}$ containing $\left\{[j, k]^{d} \mid j, k \in I^{\prime}\right\}$ and let $K_{\infty}=\{1\}$. Also let $\mathscr{K}_{d}$ denote the variety with $\rho\left(K_{d}\right)=\rho_{K_{d}}(d=1,2, \ldots, \infty)$.

REMARK 7.5. It is evident, for $N \in \mathfrak{T}$, that $K_{d} \subseteq N_{p}$ if and only if $d(N)$ divides $d$, with the obvious convention that every integer divides $\infty$.

Notation 7.6. For any positive integer $r$, let $A_{r}$ (respectively, $B_{r}$ ) denote the fully invariant subgroup of $F_{p}$ corresponding to the variety of abelian groups (respectively, all groups) satisfying $x^{r}=1$. Also let $A_{\infty}=F_{p}^{\prime}$.

LEMMA 7.7. For any positive integer $d$, or $d=\infty, \mathscr{K}_{d}$ is the largest variety with depth $d$.

Proof. By the definition of $\mathscr{K}_{d}$ and Remark 7.5 , it is clear that $\mathscr{K}_{d}$ contains any variety of depth $d$. So it remains to show that $\mathscr{K}_{d}$ itself has depth $d$.

By Remark 7.5, $d\left(\mathscr{K}_{d}\right)$ divides $d$. By the remark following Definition 2.7, $B_{d} \in \Re_{p}$ and clearly $K_{d} \subseteq B_{d}$. Thus $d\left(B_{d}\right)$ divides $d$. However, if $j, k \in I^{\prime}$, then clearly $[j, k]^{r} \notin B_{d}$ for any positive integer $r<d$. Hence $d\left(B_{d}\right)=d$. Since $K_{d} \subseteq B_{d}, d\left(K_{d}\right) \geqslant d\left(B_{d}\right)=d$ and so $d\left(\mathscr{K}_{d}\right)=d\left(K_{d}\right)=d$.

LEMmA 7.8. If $N \in \mathfrak{R}$ is such that $d(N)=d$, then $N_{p} F_{p}^{\prime}=A_{d}$.

Proof. By Proposition 5.2, $N_{p} F_{p}^{\prime}$ is a fully invariant subgroup of $F_{p}$. Since it contains $F_{p}^{\prime}$, it must be of the form $A_{r}$, for some $r$.

If $d=\infty$, then $N_{p} F_{p}^{\prime} \supseteq F_{p}^{\prime}=A_{\infty}$. For $d<\infty, N_{p} F_{p}^{\prime}$ contains $F_{p}^{\prime}$ and the elements $[j, k]^{d}$ for $j, k \in I^{\prime}$. But $A_{d}$ is the smallest fully invariant subgroup of $F_{p}$ containing $F_{p}^{\prime}$ and the elements $[j, k]^{d}$ for $j, k \in I^{\prime}$. Hence $A_{d} \subseteq N_{p} F_{p}^{\prime}$, in both cases, and $r$ must divide $d$.

Suppose that $A_{d} \neq N_{p} F_{p}^{\prime}$. Then $r<d$. Also, for $j, k \in I^{\prime},[j, k]^{r} \in A_{r}=N_{p} F_{p}^{\prime}$. Let $[j, k]^{r}=n c$, where $n \in N_{p}$ and $c \in F_{p}^{\prime}$. Then

$$
n=[j, k]^{r} c^{-1} \text {. }
$$

Let $\theta$ be the endomorphism of $F_{p}$ defined on the generators by

$$
\left[j^{\prime}, k^{\prime}\right] \theta= \begin{cases}{[j, k]} & \text { if }\left[j^{\prime}, k^{\prime}\right]=[j, k] \\ 1 & \text { otherwise. }\end{cases}
$$


Since $N$ is closed under almost complete deletion, see (2), $n \theta \in N_{p}$ where $n \theta=[j, k]^{r}(c \theta)^{-1}$. Here $c \theta$ is a word in $F_{p}^{\prime} \theta$. Now $F_{p}^{\prime} \theta$ is the derived subgroup of the subgroup of $F_{p}$ generated by $[j, k]$ and so is necessarily abelian. Hence $c \theta=1$. Thus

$$
[j, k]^{r}=n \theta \in N_{p} .
$$

But this contradicts the fact that $N$ has depth $d$. Therefore, $A_{d}=N_{p} F_{p}^{\prime}$.

Proposition 7.9. For $\mathfrak{T} \in \mathcal{L}(\mathcal{S})$, the varieties $\mathfrak{V}$ and $\mathfrak{V} \cap \mathcal{C}$ have the same depth.

Proof. If $\Re \mathscr{B} \nsubseteq \mathfrak{V}$, then $\mathscr{V} \subseteq \Re \mathcal{Q} \subseteq \mathcal{C}$ so that $\mathscr{V}=\mathscr{V} \cap \mathcal{C}$ and the result holds. So suppose that $\Re \mathscr{B} \subseteq \mathfrak{V}$, that $\rho(\mathscr{V})=\rho_{N}$ and $\rho(\mathcal{V} \cap \mathcal{C})=\rho_{M}$. Let $d(\mathscr{V})=d$. Then $M=N\left[F_{Z}, F_{p}\right]$ and so, applying $\pi_{p}$, we have $M_{p}=N_{p} F_{p}^{\prime}$. By Lemma 7.8, $M_{p}=A_{d}$ from which it follows that $d(M)=d=d(N)$ so that $d(\mathscr{V})=d(\mathscr{V} \cap \mathcal{C})$.

Notation 7.10. For any $\mathfrak{V} \in \mathcal{L}(\mathcal{C S})$, let

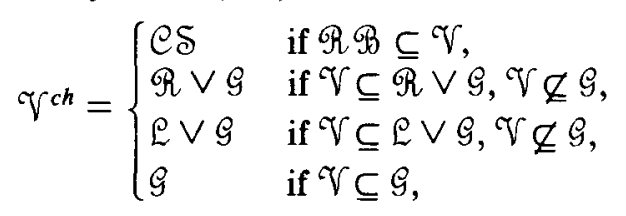

and

$$
\mathscr{V}^{*}= \begin{cases}\mathscr{V}^{c h} \cap \overline{\mathcal{V}} \cap \mathcal{K}_{d(\mathscr{V})} & \text { if } \Re \mathscr{B} \subseteq \mathfrak{T}, \\ \mathfrak{V}^{c h} \cap \overline{\mathcal{V}} & \text { otherwise. }\end{cases}
$$

It follows immediately from the definitions of $\mathfrak{V}^{c h}, \overline{\mathcal{V}}$ and Lemma 7.7, that $\mathfrak{T} \subseteq \mathfrak{V}^{*}$

LEMMA 7.11. For any $\mathfrak{V} \in \mathfrak{L}(\mathcal{C S}), \mathfrak{V} *=(\mathfrak{V} \cap \mathcal{C})^{*}$

PRoof. If $\Re \mathscr{B} \nsubseteq \mathscr{T}$, then $\mathcal{V} \subseteq \mathcal{C}$ and the result follows trivially. So let $\Re \mathscr{B} \subseteq \mathcal{T}$, $\rho(\mathcal{V})=\rho_{N}$, where $N \in \mathfrak{R}$, and $d=d(\mathscr{V})$. First $\mathfrak{V}^{c h}=\mathcal{C S}=(\mathfrak{V} \cap \mathcal{C})^{c h}$. Then

$(\mathfrak{V} \cap \mathcal{C}) \cap \mathcal{G}=\mathscr{V} \cap(\mathcal{C} \cap \mathcal{G})=\mathscr{V} \cap \mathcal{G}$

so that $\overline{\mathscr{V} \cap \mathcal{C}}=\overline{\mathscr{V}}$. Finally, by Proposition 7.9, $d(\mathscr{V} \cap \mathcal{C})=d(\mathscr{V})$. Hence

$$
\mathfrak{V}^{*}=\mathscr{V}^{c h} \cap \overline{\mathscr{V}} \cap \mathscr{K}_{d(\mathscr{V})}=(\mathfrak{V} \cap \mathcal{C})^{c h} \cap \overline{(\mathfrak{V} \cap \mathcal{C})} \cap \mathcal{K}_{d(\mathscr{V} \cap \mathcal{C})}=(\mathfrak{V} \cap \mathcal{C})^{*}
$$

Proposition 7.12. Let $($ ข, $) \in \mathcal{L}(\mathcal{C}) \times[\mathcal{C}, \mathcal{C}]$ be such that $\circlearrowleft \subseteq \mathcal{Q} \vee \vee C$. Let $\mathfrak{V}=\mathcal{Q}^{*} \cap \mathcal{W}$. Then

$$
\mathfrak{V} \cap \mathcal{e}=\mathcal{Q} \text { and } \mathfrak{v} \vee \mathcal{e}=\mathscr{w}
$$


Proof. We have

$$
\begin{aligned}
\mathscr{\mathcal { C }} & =\left(\mathscr{Q}^{*} \cap \mathscr{W}\right) \vee \mathcal{C} \\
& =\left(\mathcal{U}^{*} \vee \mathcal{C}\right) \cap(\mathscr{\mathcal { W }} \vee \mathcal{C}) \text { by Corollary } 6.6, \\
& =\left(\mathscr{U}^{*} \vee \mathcal{C}\right) \cap \mathscr{W} \\
& =\mathscr{W} \text { by hypothesis, }
\end{aligned}
$$

establishing the second claim.

If $d=d(\mathcal{Q})$, then

$$
\begin{aligned}
\mathscr{V} \cap \mathcal{C} & =\mathcal{Q}^{*} \cap \mathcal{W} \cap \mathcal{e}=\mathcal{Q}^{*} \cap \mathcal{C} \\
& =\mathcal{Q}^{\text {ch }} \cap \overline{\mathcal{Q}} \cap \mathcal{K}_{d} \cap \mathcal{C} .
\end{aligned}
$$

By the definitions of the varieties $\mathcal{Q}^{c h}$ and $\overline{\mathcal{U}}, \mathcal{Q} \subseteq \mathcal{Q}^{c h} \cap \overline{\mathcal{Q}}$; by Lemma 7.7, $\mathcal{Q} \subseteq K_{d}$ and by assumption, $\mathcal{U} \subseteq \mathcal{C}$. Thus

$$
\text { U } \subseteq \mathfrak{T} \cap \mathcal{C} \text {. }
$$

Furthermore, $\mathscr{Q} \cap \mathcal{G} \subseteq(\mathfrak{V} \cap \mathcal{C}) \cap \mathcal{G} \subseteq \overline{\mathcal{Q}} \cap \mathcal{S}=\mathscr{Q} \cap \mathcal{G}$ so that

$$
\mathscr{Q} \cap \mathcal{G}=(\mathfrak{V} \cap \mathcal{C}) \cap \mathcal{G} \text {. }
$$

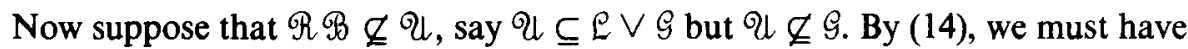
$\mathfrak{V} \cap \mathcal{E} \subseteq \mathcal{E}$ but $\mathscr{V} \cap \mathcal{E} \notin \mathcal{G}$. Hence $\mathscr{Q}$ and $\mathcal{V} \cap \mathcal{C}$ are varieties of left groups not contained in $\mathcal{G}$ but containing the same groups. Consequently, $\mathscr{Q}=\mathfrak{V} \cap \mathcal{C}$. A similar argument will establish equality if $\mathscr{Q} \subseteq \Re \vee \mathcal{G}$.

So suppose that $\Re \mathscr{G} \subseteq \mathcal{U}$. Let $\rho(\mathcal{U})=\rho_{M}$ and $\rho(\mathfrak{V} \cap \mathcal{C})=\rho_{N}$, where $M, N$ $\in \mathfrak{X}$. In view of (15), it remains to show that $M \subseteq N$. From (14) and Propositions 2.14 and 2.17 ,

$$
N=\{1\} N_{q}\left(F_{Z}\right) K_{d}\left[F_{Z}, F_{p}\right] .
$$

By (16) and Theorem 2.12,

$$
M_{q}=N_{q} .
$$

Since $\mathcal{Q} \in \mathcal{L}(\mathcal{C})$, it follows from Proposition 2.17, that

$$
\left[F_{Z}, F_{p}\right] \subseteq M
$$

so that $F_{p}^{\prime} \subseteq M_{p}$. Hence, by Proposition 5.2, $M_{p}$ is fully invariant and consequently $M_{p}=A_{r}$, for some $r$. But then $r=d(M)=d(\mathcal{Q})=d$. Thus $M_{p}=A_{d}$. We have from (17) that $N_{p} \supseteq K_{d} F_{p}^{\prime}$, where $K_{d} F_{p}^{\prime}$ is a fully invariant subgroup of $F_{p}$ by Proposition 5.2. Consequently, $K_{d} F_{p}^{\prime} \supseteq A_{d}$ so that

$$
N_{p} \supseteq K_{d} F_{p}^{\prime} \supseteq A_{d}=M_{p} .
$$


Hence,

$$
\begin{aligned}
M & =M_{q}\left(M \cap\left[F_{Z}, F_{p}\right]^{\wedge}\right) M_{p} \quad \text { by Lemma } 6.2, \\
& =N_{q}\left[F_{Z}, F_{p}\right]^{\wedge} M_{p} \quad \text { by }(18) \text { and }(19), \\
& \subseteq N_{q}\left[F_{Z}, F_{p}\right]^{\top} N_{p} \text { by }(20), \\
& \subseteq N \text { by (17). }
\end{aligned}
$$

Therefore, $M=N$ and $\mathscr{Q}=\mathscr{V} \cap \mathcal{C}$, as required.

We now combine the last corollary of the preceding section with the principal results of this section in the main result of the last three sections as follows.

THEOREM 7.13. The mapping

$$
\eta: \mathfrak{V} \rightarrow(\mathfrak{V} \cap \mathcal{e}, \mathfrak{V} \vee \mathcal{C}) \quad(\mathfrak{V} \in \mathcal{e}(\mathcal{C S}))
$$

is an isomorphism of $\mathrm{E}(\mathrm{eS})$ onto the subdirect product

$$
\mathscr{P}=\{(\mathcal{Q}, \mathscr{W}) \in \mathcal{E}(\mathcal{C}) \times[\mathcal{C}, \mathcal{C} S] \mid \mathcal{W} \subseteq \mathcal{Q} \vee \mathcal{C}\}
$$

Moreover, for any $\mathscr{V} \in \mathcal{L}(\mathcal{S})$ and ( $\mathcal{Q}, \mathscr{W})$ in this subdirect product,

$\mathfrak{V} \cap \mathcal{C}=\mathscr{q}, \mathfrak{V} \vee \mathcal{C}=\mathscr{W} \Leftrightarrow \mathfrak{V}=\mathcal{Q} * \cap \mathscr{W}$

PROOF. The homomorphism property follows directly from Corollary 6.6 , as does the fact that $\eta$ is one-to-one.

For any $\mathscr{V} \in \mathcal{L}(\mathcal{C S}), \mathcal{T} \subseteq \mathcal{V}^{*}$ while also $\mathcal{V}^{*}=(\mathscr{V} \cap \mathcal{C})^{*}$ by Lemma 7.11. Thus

$$
\mathfrak{V} \vee \mathcal{C} \subseteq \mathfrak{T} * \mathcal{e}=(\mathfrak{V} \cap \mathcal{e}) * \vee \mathcal{C}
$$

so that $\mathscr{V} \eta \in \mathscr{P}$. Conversely, for any $(\mathcal{Q}, \mathcal{W}) \in \mathscr{P}$, let $\mathcal{V}=\mathcal{Q}^{*} \cap \mathcal{W}$. By Proposition $7.12, \mathcal{T} \eta=(\mathcal{U}, \mathscr{W})$ so that $\eta$ maps $\mathcal{L}(\mathcal{C S})$ onto $\mathscr{P}$.

The direct part of the final claim follows from Proposition 7.12 and the fact that $\eta$ is one-to-one while the converse part follows from Proposition 7.12.

In the final result we characterize the congruence induced by $\eta_{1}$.

Proposition 7.14. For ข, $\mathfrak{T} \in \mathfrak{L}(\mathcal{C})$,

$$
\mathscr{U} \cap \mathcal{C}=\mathfrak{V} \cap \mathcal{C} \Leftrightarrow \mathcal{U}^{c h}=\mathscr{V}^{c h}, \mathscr{Q} \cap \mathcal{G}=\mathfrak{V} \cap \mathcal{G} \text { and } d(\mathscr{Q})=d(\mathfrak{V})
$$

Proof. Let $\mathscr{Q} \cap \mathcal{C}=\mathscr{V} \cap \mathcal{C}$. It is easily seen from this that $\mathscr{Q}^{c h}=\mathfrak{V}^{c h}$. Further

$$
\begin{aligned}
\mathscr{U} \cap \mathcal{S} & =\mathscr{Q} \cap(\mathcal{C} \cap \mathcal{G})=(\mathscr{U} \cap \mathcal{C}) \cap \mathcal{G}=(\mathfrak{V} \cap \mathcal{C}) \cap \mathcal{S} \\
& =\mathscr{V} \cap(\mathcal{C} \cap \mathcal{G})=\mathscr{V} \cap \mathcal{G} .
\end{aligned}
$$


Finally, by Proposition 7.9,

$$
d(\mathcal{Q})=d(\mathcal{Q} \cap \mathcal{C})=d(\mathscr{V} \cap \mathcal{C})=d(\mathscr{V})
$$

Conversely, suppose that $\mathscr{Q}^{c h}=\mathfrak{V}^{c h}, \mathscr{Q} \cap \mathcal{G}=\mathfrak{V} \cap \mathcal{G}$ and $d(\mathscr{Q})=d(\mathscr{V})=d$, say. In addition, let us assume for the moment that $\Re \mathscr{R} \subseteq \mathcal{Q} \cap \mathcal{V}$. Let $\rho(\mathcal{Q})=$ $\rho_{M}, \rho(\mathscr{V})=\rho_{N}$ where $M, N \in \mathcal{X}$. Then $\rho(\mathcal{Q} \cap \mathcal{C})=\rho_{M\left[F_{Z}, F_{p} \hat{\mid}\right.}$ and $\rho(\mathcal{V} \cap \mathcal{C})=$ $\rho_{N\left[F_{Z}, F_{p}\right]} \hat{\text {. }}$

Since $\mathcal{Q} \cap \mathcal{G}=\mathfrak{\mathcal { V }} \cap \mathcal{G}$, we have from Theorem 2.12 that

$$
M_{q}=N_{q} \text {. }
$$

By Lemma 7.8,

$$
M_{p} F_{p}^{\prime}=A_{d}=N_{p} F_{p}^{\prime}
$$

Hence

$$
\begin{aligned}
& M\left[F_{Z}, F_{p}\right]^{\wedge}=M_{q}\left(M \cap\left[F_{Z}, F_{p}\right]^{n}\right) M_{p}\left[F_{Z}, F_{p}\right]^{\wedge} \quad \text { by Lemma 6.2, } \\
& =M_{q}\left(M \cap\left[F_{Z}, F_{p}\right]^{\wedge}\right)\left[F_{Z}, F_{p}\right]^{\wedge} M_{p} \\
& =M_{q}\left[F_{Z}, F_{p}\right]^{\wedge} M_{p} \\
& =N_{q}\left[F_{Z}, F_{p}\right]^{\wedge} M_{p} \text { by }(21) \text {, } \\
& =N_{q}\left[F_{Z}, F_{p}\right]^{\wedge} F_{p}^{\prime} M_{p} \text { since } F_{p}^{\prime} \subseteq\left[F_{Z}, F_{p}\right]^{\wedge} \text {, } \\
& =N_{q}\left[F_{Z}, F_{p}\right]^{\wedge} F_{p}^{\prime} N_{p} \text { by }(22) \text {, } \\
& =N_{q}\left[F_{Z}, F_{p}\right]^{\wedge} N_{p} \\
& =N_{q}\left(N \cap\left[F_{Z}, F_{p}\right]^{\wedge}\right) N_{p}\left[F_{Z}, F_{p}\right]^{\wedge} \\
& =N\left[F_{Z}, F_{p}\right]^{\wedge} \quad \text { by Lemma 6.2. }
\end{aligned}
$$

Therefore $\rho(\mathcal{Q} \cap \mathcal{C})=\rho(\mathcal{V} \cap \mathcal{C})$ so that $\mathcal{Q} \cap \mathcal{C}=\mathscr{V} \cap \mathcal{C}$.

Suppose now that it is not the case that $R \mathscr{B} \subseteq \mathcal{Q} \cap \mathcal{V}$. Since $\mathcal{U}^{c h}=\mathcal{V}^{c h}$, it follows that both $\Re \Re \nsubseteq \mathscr{Q}$ and $\Re \mathscr{B} \nsubseteq \mathcal{V}$ and that $\mathscr{Q}$ is a variety of groups (respectively, left groups or right groups) if and only if $\mathfrak{V}$ is a variety of groups (respectively, left groups or right groups). Combining this with the observation that $\mathscr{Q} \cap \mathcal{G}=\mathscr{V} \cap \mathcal{G}$ we see that, in these cases, we must have $\mathscr{Q}=\mathscr{V}$ and so $\mathcal{U} \cap \mathcal{e}=\mathfrak{v} \cap \mathcal{e}$.

COROLlary 7.15. For $\mathcal{Q}, \mathcal{V} \in \mathcal{L}(\mathcal{C S})$,

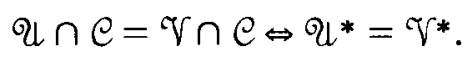

Proof. This follows immediatly from the definition of $\mathcal{U}^{*}$ and $\mathcal{V}^{*}$, Proposition 7.14 and the observation that

$$
\mathscr{Q} \cap \mathcal{G}=\mathfrak{V} \cap \mathcal{G} \Leftrightarrow \overline{\mathcal{Q}}=\overline{\mathscr{V}} .
$$




\section{References}

[1] G. Birkhoff, Lattice theory, 3rd ed., Amer. Math. Soc. Colloquium Publications, 25, Providence, R. I., 1967.

[2] A. H. Clifford, 'The free completely regular semigroup', J. Algebra 59 (1979), 434-451.

[3] T. E. Hall and P. R. Jones, 'On the lattice of varieties of bands of groups', Pacific J. Math 91 (1980), 327-337.

[4] J. M. Howie, An introduction to semigroup theory (Academic Press, London, 1976).

[5] P. R. Jones, 'Completely simple semigroups: free products, free semigroups and varieties', Proc. Roy. Soc. Edinburgh Sect. A 88 (1981), 291-313.

[6] M. Petrich, Introduction to semigroups (Merrill, Columbus, 1973).

[7] M. Petrich and N. R. Reilly, 'Varieties of groups and of completely simple semigroups', Bull. Austral. Math. Soc. 23 (1981), 339-359.

[8] M. Petrich and N. R. Reilly, 'Near varieties of idempotent generated completely simple semigroups', Algebra Universalis, to appear.

[9] M. Petrich and N. R. Reilly, 'All varieties of central completely simple semigroups', Trans. Amer. Math. Soc. 280 (1983), 623-636.

[10] V. V. Rasin, 'Free completely simple semigroups', Ural. Gos. Univ. Mat. Zap. 11 (1979), 140-151 (Russian).

\section{Simon Fraser University}

Burnaby, British Columbia

Canada 